\title{
O ENSINO DE ENFERMAGEM EM SAÚDE MENTAL NAPERCEPÇÃO DE ESTUDANTES
}

Aline Mesquita Lemos ${ }^{1}$

Helder de Pádua Lima²

Maria Salete Bessa Jorge ${ }^{1}$

Lourdes Suelen Pontes Costa ${ }^{1}$

Maria Raquel Rodrigues Carvalho'

Emilia Carvalho Caminha ${ }^{1}$ https://orcid.org/0000-0002-9242-6580 https://orcid.org/0000-0002-3795-6343

https://orcid.org/0000-0001-6461-3015

https://orcid.org/0000-0001-8589-0214

https://orcid.org/0000-0002-0194-8851

https://orcid.org/0000-0003-3098-6563

Objetivo: Compreender a experiência de estudantes de Enfermagem no processo de ensino-aprendizagem em saúde mental. Métodos: Estudo qualitativo, realizado com oito estudantes de enfermagem de uma instituição pública. As narrativas foram coletadas através de grupo focal, gravadas e transcritas na íntegra. A análise pautou-se na técnica de análise categorial temática. Resultados: Emergiram duas categorias analíticas: Formação em saúde mental na Graduação em Enfermagem e Experiências dos estudantes no processo de ensino-aprendizagem. Conclusão: A experiência discente apontou: a necessidade de potencializar a formação em saúde mental com a criação de disciplinas que abordassem esta área de conhecimento; desenvolvimento de estratégias de integração do conteúdo deste campo específico com o de outras disciplinas; uso de metodologias ativas e inovadoras; maiores oportunidades de atuação na Rede de Atenção Psicossocial; e desenvolvimento de práticas de cuidado em enfermagem que favoreçam a superação da estigmatização da pessoa em sofrimento psíquico.

Descritores: Enfermagem, Saúde mental, Estudantes de enfermagem, Educação em enfermagem, Enfermagem psiquiátrica.

\section{MENTAL HEALTH NURSING TEACHING BASED ON STUDENTS' PERCEPTION}

Objectives: Analyzing nursing students' experience in the teaching-learning process in mental health. Methods: Study qualitative conducted with eight nursing students from a public institution. Participants' narratives were recorded in a focus group and fully transcribed. Data analysis was based on the theme/category-based content analysis technique. Results: Two analytical categories emerged, namely: Mental Health Training in Undergraduate Nursing and Students experiences in teaching-learning process. Conclusion: Students' experience pointed towards the need of improving mental health training by creating a discipline focused on addressing this knowledge field; developing strategies focused on integrating the content of this specific field with that of other disciplines; using active and innovative methodologies; enabling greater opportunities for students to act in the Psychosocial Care Network; and developing nursing care practices to help individuals in psychological distress to overcome stigmatization.

Descriptors: Nursing, Mental health, Students nursing, Education nursing, Psychiatric nursing.

\section{LA ENSEÑANZA DE ENFERMERÍA EN SALUD MENTAL EN LA PERCEPCIÓN DE LOS ESTUDIANTES}

Objetivo: Entender la experiencia de los estudiantes de enfermería en el proceso de enseñanza-aprendizaje en salud mental. Métodos: Un estudio cualitativo, realizado con ocho estudiantes de enfermería de una institución pública. Las narrativas fueron recopiladas a través de grupo focal, grabadas y totalmente transcritas. El análisis se basó en la técnica de análisis por categoría y tema. Resultados: Surgieron dos categorías analíticas: Formación en Salud Mental en enfermería del pregrado y Experiencias de los estudiantes en la participación en el proceso de enseñanza-aprendizaje. Conclusión: La experiencia de los estudiantes señaló la necesidad de potenciar la formación en salud mental con la creación de disciplina que aborde esta área del conocimiento; el desarrollo de estrategias de integración del contenido de este campo específico con el de otras disciplinas; el uso de metodologías activas e innovadoras; mayores oportunidades de acción en la Red de Atención Psicosocial; y el desarrollo de prácticas de cuidado en enfermería que favorezcan la superación de la estigmatización de la persona con sufrimientos psicológicos.

Descriptores: Enfermería, Salud mental, Estudiantes de enfermeira, Educación em enfermeira, Enfermería psiquiátrica.

IUniversidade Estadual do Ceará - UECE, CE, Brasil.

${ }^{2}$ Universidade Federal de Mato Grosso do Sul - UFMS, MS, Brasil.

Autor Correspondente: Aline Mesquita Lemos Email: alinemcastro@hotmail.com

Recebido: 21/1/20 Aceito: 11/4/20 


\section{INTRODUÇÃO}

Atualmente, a formação em Enfermagem alinha-se à Lei de Diretrizes e Bases da Educação Nacional e às Diretrizes Curriculares Nacionais (DCN) do Curso de Graduação em Enfermagem, as quais propõem que os currículos norteiem a construção do perfil acadêmico e profissional a partir de habilidades, competências e conhecimentos que atendam à demanda profissional do Sistema Único de Saúde (SUS) ${ }^{1-2}$.

As Universidades têm autonomia para estabelecer seus currículos de acordo com os diferentes componentes pedagógicos. As Reformas Sanitária e Psiquiátrica influenciam a estrutura curricular do curso de Enfermagem e determinam o perfil do enfermeiro para atuação em diferentes cenários do cuidado profissional ${ }^{3}$.

O ensino do cuidado aplicado à saúde mental nos Cursos de Graduação em Enfermagem está, de maneira geral, disponivel em disciplinas denominadas de Enfermagem em Saúde Mental e/ou Psiquiátrica que visam, entre outros objetivos, o desenvolver de habilidades e competências vinculadas aos princípios da Reforma Psiquiátrica e do relacionamento terapêutico, assim como o exercício constante da percepção de si próprio na relação enfermeiro/paciente 4 .

A passagem da institucionalização para a inserção social da pessoa em sofrimento psíquico exige que enfermeiros integrem saberes e práticas que permitam tais profissionais o reconheçam a presença desses pacientes em uma realidade social e também em seu contexto familiar. A habilidade de reconhecer tal processo durante a formação profissional requer que o ensino de atenção em saúde valorize aspectos biopsicossociais e supere o modelo biomédico e hospitalocêntrico/manicomial ${ }^{3.5}$

A formação de enfermeiros no campo da saúde mental é deficiente em função de vários fatores como: falta de definição das competências específicas; predisposição de discentes para atuar em outras áreas de interesse; particularidades das metodologias utilizadas pelo corpo docente; formação precária e desconhecimento do currículo por parte de alguns docentes; e ausência da transversalidade no tema Saúde Mental nas disciplinas voltadas ao ciclo da vida ${ }^{6}$

É essencial estender o olhar sobre o fenômeno do ensino e do aprendizado em saúde mental, não apenas acerca da ação docente mas, também, das perspectivas de estudantes de Enfermagem que compartilham essa experiência relacional, ao considerar que essa ação projetada significa para futuros enfermeiros.

A experiência dos autores como docentes da área de Enfermagem de diferentes instituições de ensino superior, comprometidos com o fortalecimento da formação de enfermeiros em saúde mental, motivou a realização desta pes- quisa, a qual foi norteada pela seguinte questão: quais são as percepções de estudantes de Enfermagem sobre o processo de ensino-aprendizagem aplicado à saúde mental?

Dessa forma, o objetivo do presente estudo é compreender a experiência de estudantes de Enfermagem no processo de ensino-aprendizagem em saúde mental.

\section{MÉTODO}

\section{Tipo de estudo}

Realizou-se um estudo exploratório com abordagem qualitativa focado na exploração de fenômenos em sua essência e em experiências vividas.

\section{Participantes da pesquisa}

A população do estudo foi representada por 21 estudantes matriculados no oitavo período do curso de Enfermagem que haviam cursado a disciplina "Enfermagem no Processo de Cuidar do Adulto II" no sexto período. Essa era a única disciplina da matriz curricular na qual o conteúdo teórico-prático de saúde mental era abordado.

A amostra foi representada por oito estudantes que atendiam aos seguintes critérios de inclusão: ter idade igual ou superior a 18 anos e estar matriculado no oitavo período do Curso de Graduação em Enfermagem e ter cursado. Foram excluidos do estudo estudantes que trancaram suas matrículas na disciplina durante a coleta de dados ou que se recusaram a participar. A amostra foi definida de acordo com o critério de satisfação dos dados, o qual permitiu a inclusão intencional dos estudantes que atendiam aos critérios de inclusão e estavam disponíveis durante a coleta de dados.

Cada informante foi identificado de acordo com a ordem de aceite para participação com o objetivo de garantir o anonimato. Dessa forma, "Pl" representa o estudante que primeiro que aceitou participar da pesquisa e assim sucessivamente.

\section{Local de estudo}

Desenvolveu-se o estudo no Curso de Enfermagem de uma Universidade Pública no Ceará, Brasil.

\section{Coleta de dados}

Um dos pesquisadores compareceu à sala de aula dos estudantes que atendiam os critérios de inclusão na pesquisa, com a autorização da coordenação do curso, disponibilizou informações sobre a pesquisa e fez um convite para a participação no estudo.

Foram definidos previamente a equipe técnica (uma mediadora e dois observadores), o local de realização e suas características (uma das salas do departamento de Enfermagem; fora do horário de aula; ambiente acolhedor, agradável e confortável; com cadeiras dispostas de forma circular para facilitar a participação e interação entre os participantes), a 
técnica de coleta e o roteiro de discussão (uma sessão de grupo focal orientada por questões norteadoras que versavam sobre o conteúdo de Saúde Mental apresentado durante Curso de Graduação em Enfermagem, o processo ensino-aprendizagem e a vivência na disciplina "Enfermagem no Processo de Cuidar do Adulto II"). A coleta de dados foi realizada em junho de 2017 e a sessão de grupo focal dividiu-se em três tempos: Apresentação, momento com duração de 15 minutos em que equipe e participantes se apresentaram, os objetivos da pesquisa e os direitos dos participantes foram esclarecidos, e o Termo de Consentimento Livre e Esclarecido foi assinado por parte daqueles que concordaram em participar; Desenvolvimento, momento com duração de 60 minutos em que se conduziu a entrevista guiada pelo roteiro de questões norteadoras, no qual a facilitadora utilizou técnicas investigativas para fomentar a discussão e os observadores registraram o andamento do grupo focal e gravaram a discussão com gravador digital; e Término, momento com duração de 15 minutos em que foram reforçados os objetivos da pesquisa $e$ os direitos dos participantes, realizados os agradecimentos e oferecido um lanche.

\section{Análise dos dados}

Os dados foram analisados a partir de práticas discursivas e da produção de sentidos ${ }^{7}$, substanciado pelas narrativas gravadas e transcritas na integra. Com base nesse tipo de análise, seguiram-se as seguintes etapas: digitou-se o material gravado no programa Word $₫$, inseriu-se um quadro com a quantidade de colunas correspondente às categorias a serem empregadas, transferiu-se o texto para as colunas por meio das ferramentas copias e colar, e realizou-se a leitura das narrativas procurando identificar os sentidos produzidos pelos estudantes acerca da formação em saúde mental na Graduação em Enfermagem e das experiências no processo de ensino-aprendizagem.

\section{Aspectos éticos}

O estudo foi aprovado pelo Comitê de Ética em Pesquisa, sob Protocolo $n^{\circ} 480.404$. Todos os aspectos éticos e legais descritos na Resolução №466/2012 do Conselho Nacional de Saúde ${ }^{8}$ foram respeitados.

\section{RESULTADOS}

Todos os participantes eram mulheres, solteiras e tinham idade entre 21 e 23 anos. Seis delas eram naturais do municipio de Fortaleza - Ceará. Nenhuma participante tinha filhos. Seis delas eram bolsistas de iniciação científica ou de projetos extensão. Quatro participantes tinham renda individual menor que um salário mínimo e cinco tinham renda familiar entre três e oito salários mínimos.

A seguir, serão apresentadas duas categorias ana- líticas que emergiram das narrativas: "Formação em saúde mental na Graduação em Enfermagem" e "Experiências dos estudantes no processo de ensino-aprendizagem".

Formação em saúde mental na Graduação em Enfermagem

As narrativas das estudantes revelaram a insatisfação com o conteúdo aplicado à saúde mental abordado na disciplina "Enfermagem no Processo de Cuidar do Adulto II". Tal fato resultou da carga horária reduzida, do tema ser abordado apenas no sexto período de curso, e também, da falta de integração com as demais disciplinas curriculares. Tal contexto pode ser apreendido a partir das seguintes narrativas:

Só vi conteúdo de Saúde Mental no sexto período. Acho que deveria abordar no quinto semestre mostrando o que podemos encontrar no Posto de Saúde. Fui para o Estágio Supervisionado na atenção básica sem saber nada de Saúde Mental (Pl);

É inviável ter o conteúdo de Saúde Mental somente na disciplina de Saúde do Adulto. Tem muita sobrecarga. Na grade curricular são nove semestres, você só tem a Saúde Mental nessa disciplina, que já é tão sobrecarregada... Se eles pudessem desuincular com certeza ficaria melhor para os estudantes (P8).

Outro aspecto presente nas narrativas refere-se à superficialidade da abordagem dada aos temas relativos ao campo da saúde mental e à insatisfação com a metodologia e didática utilizadas em aulas teóricas. As narrativas a seguir revelam tal cenário

Para mim, não foi suficiente esse aprendizado. A professora que deu aula sentou e leu slide. A matéria já não é legal, já tem pouca oportunidade e quando tem, não é muito boa (Pl);

Mesmo no sexto semestre foi uma coisa pincelada. A parte da teoria foi muito conceitual. Eram conceitos que eram passados prontos. Só estudávamos basicamente para as provas. Não tinha estudo de caso, nada para raciocinar, pensar um pouco mais sobre por qual motivo estava acontecendo aquilo, se estava ligado à isso ou aquilo (P6)

As narrativas mostram que a formação dos enfermeiros no campo da saúde mental, ainda é permeada de estigmas e fragilidades. Apenas uma disciplina aborda o tema e os docentes não dispõem de estratégias didáticas de ensino.

Experiências dos estudantes no processo de ensino-aprendizagem

As narrativas das estudantes mostram que foram fei- 
tas visitas e ações em diferentes serviços que integram a Rede de Atenção Psicossocial (RAPS). Neles, as alunas desenvolveram diferentes práticas de cuidado de Enfermagem aplicadas à saúde mental: relacionamento e comunicação terapêutica, coordenação de grupos, caracterização de transtornos mentais, e o cuidado individual e familiar.

As estudantes também revelam a necessidade de explorar e aprofundar a prática do Enfermeiro junto a todos os serviços da RAPS afim de romper com a formação fragmentada em Enfermagem:

No estágio no Centro de Atenção Psicossocial (CAPS) só fiz sala de espera (Pl);

Não fiz consulta, nem observei como um Enfermeiro age. Queria que cada aluno passasse um dia com um profissional, vendo o que ele fala, quais as perguntas, para usar como exemplo. Muitas vezes, ao falar com o paciente, aconselhamos o que achamos, o que temos de carga da nossa vida, nem sempre da disciplina. Vendo a atuação profissional facilitaria muito (P5);

Ficamos no Hospital-dia e depois desenvolvemos o relacionamento terapêutico com o paciente através de um instrumento. Nosso trabalho terapêutico era com o paciente e com a família também. Desenvolvi estudo de caso, mas ficou a desejar (P7);

Tinha que ler muito, querendo ou não. Se tivesse ido ao CAPS, tinha detestado todo dia fazer sala de espera. Enfermeiro não se resume a isto. Teria fugido várias vezes do estágio (P8).

As narrativas revelam que a atuação em dispositivos da RAPS favorece a aproximação com usuários do sistema e a superação do estigma imputado à pessoa em sofrimento psíquico. Outros trechos denotam que a experiência das estudantes com pacientes de saúde mental mostrou um déficit de conhecimento e pouco interesse pela área:

Atendemos pacientes em crise, ficamos desesperadas, medrosas, sem ação. Se pedir hoje para atuar na Saúde Mental, não vou saber, vou ter que estudar, não me considero capaz, minha experiência foi pouca. O conteúdo não é interessante para mim (P2);

Eu tinha muito preconceito, não sabia o que fazer em um Hospital de Saúde Mental, o que era sofrimento mental, como é que está aquela pessoa. Por exemplo, no Hospital-dia, ficar com o paciente todo dia em relacionamento terapêutico é diferente. A maioria das pessoas não tem interesse, mas o contato e uma aula podem facilitar e ser determinante (P7)
As participantes destacaram a necessidade de participar ativamente em projetos de extensão comunitária na referida área de modo a suprir deficiências de aprendizado em saúde mental na graduação:

Participei de um Projeto Integrado de Pesquisa e de Extensão. Lá observei o que é sindrome do pânico, pelo depoimento de pacientes, não pelo que eu leio em um liuro. Se me perguntar o que é eu sei dizer, pois ouvi pessoas falando. Visualizei os sintomas de depressão, pessoas em uso exacerbado de medicamentos, em terapias alternativas no tratamento (P3);

A Extensão foi o pouco da minha bagagem, do significado que tem Saúde Mental. As pessoas participauam e víamos a diferença ao longo dos encontros. Dava para ter uma continuidade da assistência (P4).

As narrativas revelam que a experiência vivida pelas participantes possibilitou sua reflexão e ampliação de conhecimento associadas às práticas em serviços e ações de saúde, embora as narrativas apontem resistência à atuação no campo da saúde mental. Nesse sentido, o ensino superior destaca-se como ferramenta para quebrar paradigmas e propagar a humanização do cuidado.

\section{DISCUSSÃO}

No Brasil, as primeiras tentativas de sistematização do ensino de Enfermagem na área psiquiátrica ocorreram a partir de 1890, na Escola Profissional de Enfermeiros no Hospício Nacional de Alienados. No ano de 1923 se institucionaliza o ensino da Enfermagem, mas não houve interesse do ensino nesta área específica. Alguns cursos iniciaram a formação em Enfermagem Psiquiátrica por volta de 1941, no contexto de expansão dos manicômios ${ }^{3}$.

Apesar de terem se tornado obrigatórios na formação em Enfermagem no país em 1949, com a promulgação da Lei № 775 e do Decreto № 27.426, o ensino e o estágio de Enfermagem Psiquiátrica se basearam, até a década de 1980, nos princípios assistenciais estabelecidos pelos paradigmas biomédico e hospitalocêntrico que tinham como foco a patologia, o tratamento medicamentoso e a reclusão de pessoas com transtornos mentais em instituições manicomiais de longa permanência?.

A partir da década de 1990, a área de conhecimento da saúde mental e psiquiatria sofreu mudanças significativas no currículo de Enfermagem para atender ao contexto histórico, político e social relacionado aos movimentos de Reforma Sanitária, que culminou com a instituição do SUS, e de Refor- 
ma Psiquiátrica que, de certa forma, determinou o perfil do profissional para atuar nessa área em diferentes cenários de cuidado profissional ${ }^{3}$.

A Política Nacional de Saúde Mental sancionada pela Lei № 10.216 em 2001, paralelamente à instituição das DCN do Curso de Graduação em Enfermagem, dispôs sobre a proteção e os direitos das pessoas portadoras de transtornos mentais e comportamentais e redirecionou o modelo assistencial em saúde mental, conforme o paradigma da atenção psicossocial. A oferta de tratamento em serviços abertos de base territorial e comunitária passou a ser prioritária ${ }^{10}$.

A consolidação dessas políticas públicas passou a requerer a formação de profissionais comprometidos com a atenção à saúde mental e capazes de romper com os paradigmas biomédico e hospitalocêntrico, compreender os determinantes psicossociais da loucura, desenvolver novas formas de pensar e agir, e reinventar modos de lidar com pessoas em sofrimento psíquico. A partir de então, o ensino de Enfermagem Psiquiátrica e de Saúde Mental deve seguir as DCN e orientar-se pelos princípios da Reforma Psiquiátrica brasileira, buscando inserir estudantes nos novos dispositivos de atenção à pessoa em sofrimento psíquico evitando mantê-los exclusivamente no âmbito hospitalario-11

Tal quadro suscitou nos docentes a necessidade de repensar o conteúdo das disciplinas do Curso de Graduação em Enfermagem e os cenários de prática para o desenvolvimento de atividades com estudantes. No entanto, o rompimento com o modelo hospitalocêntrico é complexo e exige a quebra do paradigma "medicar, conter e vigiar", apesar da criação de dispositivos substitutivos aos manicômios. O novo modelo deu lugar à construção de novas estratégias assistenciais para reabilitar e promover cuidado o efetivo $0^{6,12-13}$.

Vale ressaltar que o modelo tradicional de como se constituem as práticas de formação e de cuidar ainda não foi totalmente superado. Atualmente a assistência de caráter manicomial convive lado a lado com os novos dispositivos de assistência em saúde mental. Além disso, o processo de formação em Enfermagem ainda enfrenta dificuldades na construção de saberes voltados para o alcance da integralidade da atenção em saúde como diretriz da Constituição Brasileira de 1988 para o SUS, também prevista na Lei Orgânica de Saúde da Saúde, Lei № 8.080/199010-11. Historicamente, a formação de profissionais da saúde foi pautada pelo uso de metodologias tradicionais, fragmentadas e reducionistas. A fragmentação do saber manifestou-se nas subdivisões de cursos em períodos e em disciplinas estanques. Além disso, o processo ensino-aprendizagem ainda restringe-se, muitas vezes, à reprodução do conhecimento; o docente assume o papel de transmissor de conteúdos, e os discentes os captam e repetem de forma passiva e receptiva - tornando-se meros expectadores, sem criticidade e reflexão ${ }^{14}$

Dada a peculiaridade e relevância do cuidado de enfermagem aplicada à saúde mental, é imprescindivel desenvolver estratégias inovadoras de ensino e construção de possibilidades de cuidados, tais como: relação interpessoal, projeto terapêutico, inclusão social, reabilitação psicossocial e atenção interprofissional, territorializada e intersetorial. É essencial basear o processo de formação de profissionais enfermeiros em uma pedagogia centrada em valorizar o estudante como sujeito do aprendizado e em construir conhecimento por meio de atividades e práticas interativas. Metodologias ativas são úteis nesse contexto, pois protagonizam o processo de formação acadêmica e estimulam a participação ativa de discentes no processo dinâmico de construção do conhecimento, e de resolução e avaliação de problemas ${ }^{2,6,15}$. Várias fragilidades são observadas na formação de enfermeiros em saúde mental e evidenciadas na literatura e nas narrativas das participantes do estudo. Algumas dessas fragilidades estão ligadas à aplicação da integralidade durante a formação acadêmica e do reconhecimento da Sistematização da Assistência de Enfermagem (SAE) e do processo de enfermagem, o qual é um instrumento importante de trabalho para o enfermeiro, por estar peculiarmente atrelado à prática da clínica ampliada $^{11-16}$.

Experiências que utilizam e avaliam a eficácia de tal abordagem na formação em saúde mental são limitadas. Em geral, professores oportunizam a execução de parte da abordagem discutida aqui. Ocasiões que têm como objetivo desenvolvê-la em sua totalidade restringem-se a experiências localizadas, em um contexto institucional particular, ou em estudos de caso focados em patologias $^{17}$.

O estigma imposto à pessoa em sofrimento psíquico é outra barreira relevante para o processo de ensino-aprendizagem. Esta afirmação reverbera nas escolhas feitas por esses profissionais, uma vez que a área de saúde mental não é vista como opção de ação na prática profissional por muitos estudantes na área de enfermagem.

Estigma e medo podem contribuir para que estudantes se distanciem do paciente. A formação em enferma- 
gem pode demarcar um espaço para a apropriação de como eles percebem, reagem e redimensionam do olhar sobre a clientela, priorizando práticas mais integradas na provisão de cuidados de saúde $\mathrm{e}^{18-20}$.

O contato com temas pertinentes à saúde mental durante a graduação pode ser o ponto de partida para abordar comportamentos discriminatórios que reforçam o estigma. A inserção de discentes nos serviços de assistência a pessoas em sofrimento psíquico possibilita a diminuição do preconceito e o aumento da disponibilidade para a prestação de cuidado livre de julgamentos. $\bigcirc$ enfrentamento do estigma imposto à pessoa em sofrimento psíquico é um dos desafios apontados pela Reforma Psiquiátrica, uma vez que associa-se à negação de direitos humanos dos portadores de transtornos mentais, fato que traz mais sofrimento a eles ${ }^{13,21-22}$.

As premissas das DCN aplicadas à Graduação de Enfermagem estabelecem que a formação do enfermeiro deve ser pautada no direcionamento para o SUS, dadas as necessidades sociais, com ações integradas ${ }^{1}$. $O$ incentivo da parceria com instituições nos diversos níveis de atenção dentro da assistência em saúde mental, assim como a estágios extracurriculares em outros dispositivos favorecem o processo ensino-aprendizagem de base sólida ${ }^{23}$

\section{Limitações do estudo}

O estudo apresenta limitações quanto à sua abrangência, pois restringiu-se a uma única instituição de ensino. Além disso, os dados foram coletados em uma única sessão de grupo focal e o número de participantes foi reduzido.

\section{Contribuição do estudo para a prática}

O estudo contribui para a ampliação do debate acerca da formação de enfermeiros para o cuidado em saúde mental na perspectiva exposta na Reforma Psiquiátrica. Propicia também condições para a reflexão acerca do papel dos discentes na promoção da saúde mental, bem como da necessidade de proporcionar uma formação que possibilite o desenvolvimento de habilidades científicas, humanísticas e técnicas aos estudantes de modo a instrumentalizá-los para a prática profissional embasada nas DCN e nos princípios do SUS.

\section{CONCLUSÃO}

A experiência discente apontou a necessidade de potencializar o processo de ensino-aprendizagem em saúde mental por meio de disciplinas que abordem exclusivamente tópicos dessa área de conhecimento. O desenvolvimento de estratégias de integração curricular e o uso de metodologias ativas e inovadoras que viabilizem a partilha de saberes e práticas entre estudantes e docentes também são aspectos a serem considerados.

Além dos fatores supracitados, a oportunidade de atuação nos diversos dispositivos da RAPS e de aproximação entre estudantes e usuários; o uso da SAE como referencial para intervenções em saúde mental; o estímulo e ampliação da participação discente em projetos de pesquisa e extensão comunitária para minimizar lacunas do ensino e possibilitar novas formas de construir saúde; e desenvolvimento de práticas de cuidado em enfermagem baseadas no relacionamento e na comunicação terapêutica visando superar o estigma da pessoa em sofrimento psíquico também são decisivos.

Há a expectativa de que dados do presente estudo possam instigar o interesse da comunidade científica pelo desenvolvimento de pesquisas que aprofundem o conhecimento acerca do processo ensino-aprendizagem em saúde mental. Tal abordagem deve ser substanciada pela perspectiva dos diferentes atores envolvidos no processo. Novos estudos devem produzir subsídios para alinhar projetos pedagógicos nos Cursos de Graduação em Enfermagem e o exercício da prática profissional nesse campo de estudo.

CONTRIBUIÇÔES DOS AUTORES: Aline Mesquita Lemos: concepção do estudo, análise e intepretação dos resultados, redação do artigo e revisão crítica do conteúdo intelectual, aprovação da versão final do artigo. Maria Salete Bessa Jorge: concepção do estudo, análise e intepretação dos resultados, redação do artigo e revisão crítica do conteúdo intelectual, aprovação da versão final do artigo. Helder de Pádua Lima: análise e intepretação dos resultados, redação do artigo e revisão crítica do conteúdo intelectual, aprovação da versão final do artigo. Lourdes Suelen Pontes Costa: análise e intepretação dos resultados, redação do artigo e revisão crítica do conteúdo intelectual, aprovação da versão final do artigo. Maria Raquel Rodrigues Carvalho: redação do artigo e revisão crítica do conteúdo intelectual, aprovação da versão final do artigo. Emília Carvalho Caminha: redação do artigo e revisão crítica do conteúdo intelectual, aprovação da versão final do artigo.

Agradecimentos e Financiamento: Agradecemos o apoio/financiamento da Coordenação de Aperfeiçoamento de Pessoal de Nivel Superior (CAPES). 


\section{REFERÊNCIAS}

1- Ministério da Educação. (BR). Conselho Nacional de Educação. Câmara de Educação Superior. Resolução CNE/CES n. 3, de 7 de novembro de 2001. Institui as diretrizes curriculares nacionais do curso de graduação em enfermagem. Diário Oficial da República Federativa do Brasil. Brasilia (DF), 9 de novembro de 2001. Seção 1, p.37. Available from:

2-SiqueiraJúniorAC, OtaniMAP. Theteaching of psychiatricnursing and mental health in the curriculum by competence. Rev Min Enferm [Internet]. 2011 [cited 2019 mar 20]: 15(4):539-545. Available from: http://www.scielo.br/scielo.php?script=sci_arttext\&pid=S0104-07072013000200016 3- Rodrigues J, Kempfer SS, Lenz JR, Oliveira SN. Influence of curricular reforms in mental health nursing education between 1969 and 2014. Rev Gaúcha Enferm [Internet]. 2017 [cited 2019 abr 20]; 38(3):1-9. Available from: http://www.scielo.br/scielo.php?script=sci_ arttextEpid=S1983-14472017000300407.

4- Rodrigues J, Lazzari DD. Martini JG. Testoni AK. Professors perception of mental health teaching in nursing. Texto Contexto-Enferm [Internet]. 2019 [cited 2019 jun 18]: 28(2):1-11. Available from: http://dx.doi.org/10.1590/1980-265X-TCE-2017-0012.

5- Vargas D. Maciel MED, Bittencourt MN, Lenate JS, Pereira CF. Teaching psychiatric and mental health nursing in Brazil: Curricular analysis of the undergraduation course. Texto Contexto-Enferm [Internet]. 2018 [cited 2019 jun 18]; 27(2):11-19. Available from: http://www.scielo.br/scielo. php?script=sci_arttext\&pid=S0104-07072018000200316.

6- Santos LM, Oliveira RMP, Dutra VFD, Porto IS. The process of knowledge transference: a matter concerning of teaching of psychiatric nursing. Esc Anna Nery Rev Enferm [Internet]. 2017 [cited 2019 abr 20]; 21(3):1-8. Available from: http://www.scielo.br/pdf/ean/v2ln3/14148145-ean-2177-9465-EAN-2016-0356.pdf.

7- Spink MJ. Práticas discursivas e produção de sentidos no cotidiano: aproximações teóricas e metodológicas. São Paulo: Cortez; 2013.

8- Ministério da Saúde. (BR). Conselho Nacional de Saúde. Resolução n. 466, de 12 de dezembro de 2012. Dispõe sobre diretrizes e normas regulamentadora de pesquisas envolvendo seres humanos. Diário Oficial da República Federativa do Brasil (DF), 12 de dezembro de 2012. Seção 1, p.59. Available from :

9. Martins GCS, Peres MAA, Santos TCF, Queirós PJP, Paiva CF, Almeida Filho AJ. Teaching undergraduate nursing in mental health as allied to the consolidation of the Psychiatric Reform movement. Esc Anna Nery [Internet]. 2018 [cited 2019 jul 20]; 22(4):1-8. Available from: https:// dx.doi.org/10.1590/2177-9465-ean-2018-0164

10. Queiroz AM, Florêncio RMS, Fernandes JD, Teixeira E, Silva GTR, Amestoy SC. (In)visibility of mental health at the National Seminar on Nursing Education Guidelines. Rev Enferm UERJ [Internet]. 2018 [cited 2020 abr 10]; 26(e31292):1-7. Available from: https://www.e-publicacoes. uerj.br/index.php/enfermagemuerj/article/view/31292/26830.

11- Tavares CM, Mesquita LM. Systematization of nursing and clinical assistance expanded: challenges for mental health education. Enferm Foco [Internet]. 2019 [cited 2020 mar 28]: 10(7):121-126. Available from: http://revista.cofen.gov.br/index.php/enfermagem/article/view/2810/560. 12- Souza MCBM. The Psychiatric nursing/mental health education: Advances, limitations and challenges. Rev Eletrônica Saúde Mental Alcool Drog [Internet]. 2016 [cited 2019 jul 20]; 12(3):139-146. Available from: http://www.revistas.usp.br/smad/article/view/120777/117844.

13- Tavares CMM, Gama LN, Souza MMT, Paiva LM, Silveira PG, Mattos MMGR. Specific skills of mental health nurses in undergraduate nursing teaching. Rev Port Enferm Saúde Mental [Internet]. 2016 [cited 2019 jul 20]; 4(1):25-32. Available from: http://www.scielo.mec.pt/scielo. php?script=sci_arttext\&pid=S1647-21602016000400004\&lng=pt\&nrm=iso\&tlng=pt.

14- Mitre SM, Batista RS, Mendonça JMG, Pinto NMM, Meirelles CAB, Porto CP et al. Active teaching-learning methodologies in health education: current debates. Cien Saude Colet [Internet]. 2018 [cited 2019 jun 18]; 13(2):2133-2144. Available from: http://dx.doi.org/10.1590/ S1413-81232008000900018.

15- Fernandes MA, Soares NSA, Ribeiro IAP, Sousa CCM, Ribeiro HKP. Active methodologies as a tool for training in mental health. Revenferm UFPE on line [Internet]. 2018 [cited 2019 jun 18]; 12(12):3172-3180. Available from: https://doi.org/10.5205/1981-8963-v12i12a237762p3172-3180-2018. 16. Santos ATS, Oliveira CB, Passos MC, Andrade ASA, Gallotti FCM. Integrality of care in the training of nurses: visions and experiences of nursing students. Enferm Foco [Internet]. 2019 [cited 2020 mar 28]: 10(1):122-126. Available from: http://revista.cofen.gov.br/index.php/ enfermagem/article/view/1397/507.

17. Garcia APRF, Freitas MIP, Lamas JLT, Toledo VP. Nursing process in mental health: an integrative literature review. Rev Bras Enferm [Internet] 2017 [cited 2019 jul 20]; 70 (1): 209-218. Available from: http://dx.doi.org/10.1590/0034-7167-2016-0031.

18- Azevedo AL, Araújo STC, Vidal VLL. How nursing students perceive communication with patients in mental health. Acta Paul Enferm [Internet]. 2015 [cited 2019 jul 21]; 28(2):125-131. Available from: http://www.scielo.br/scielo.php?pid=S0103-21002015000200125\&script=sci_ arttext\&tlng=en.

19-Vijayalakshmi P, Thimmaiah R, Chandra R, Badamath S. Bachelor of nursing student' attitude towards people with mental illness and career choices in psychiatric nursing. An Indian perspective. Invest Educ Enferm [Internet]. 2015 [cited 2019 jul 21]; 33(1):138-154. Available from: http://www.scielo.org.co/scielo.php?script=sci_arttext\&pid=S012053072015000100017\&lng=en\&nrm=iso.

20- Barbosa MC, Vasconcelos CR, Oselame GB. The perception of nursing on academic madness. Rev Enferm Atenção Saúde [Internet]. 2016 [cited 2019 jul 20]; 5(2):3-17. Available from: http://seer.uftm.edu.br/revistaeletronica/index.php/enfer/article/view/1516/pdf.

21- Silva LA, Santos I, Tavares CMM. Nursing students' imaginary regarding people with psychological distress: A socio-poetic study. Rev Enferm UERJ [Internet]. 2015 [cited 2018 jul 10]; 23(4):468-474. Available from: https://www.e-publicacoes.uerj.br/index.php/enfermagemuerj/ article/view/18917.

22. Martins GCS, Peres MAA, Santos TCF, Queirós PJP, Paiva CF, Almeida Filho AJ. Teaching undergraduate nursing in mental health as allied to the consolidation of the Psychiatric Reform movement. Esc Anna Nery [Internet]. 2018 [cited 2019 jul 20]; 22(4):1-8. Available from: https:// dx.doi.org/10.1590/2177-9465-ean-2018-0164.

23. Silva ANC, Moreira DP, Freitas CMA, Teixeira AKS, Pinheiro ARM. Extracurricular nursing internship: strategy for vocacional training. Enferm Foco [Internet]. 2019 [cited 2020 mar 22]; 10(4):121-135. Available from: http://revista.cofen.gov.br/index.php/enfermagem/article/view/1880. 